\title{
La construction du cerveau : de la physiologie à la physiologie, en passant par le clonage des gènes
}

\section{Constantino Sotelo}

C. Sotelo: Inserm Unité 106, Hôpital de la Salpêtrière, 47, boulevard de l'Hôpital, 75651 Paris Cedex 13, France.

\section{RÉFÉRENCES}

1. Hamburger V. Ontogeny of neuroembryology. J Neurosci 1988 ; 8 : 3535-40.

2. Puelles L, Rubenstein JL. Expression patterns of homeobox and other putative regulatory genes in the embryonic mouse forebrain suggest a neuromeric organization. Trends Neurosci $1993 ; 16: 472-9$.

3. Acampora D, Gulisano M, Simeone A. Otx genes and the genetic control of brain morphogenesis. Mol Cell Neurosci 1999; $13: 1-8$

4. Edlund T, Jessell TM. Progression from extrinsic to intrinsic signaling in cell fate specificiation : a view from the nervous system. Cell 1999; $96: 211-24$.

5. Tessier-Lavigne M, Goodman CS. The molecular biology of axon guidance. Science $1996 ; 274$ : 1123-33.

\author{
TIRÉS À PART \\ C. Sotelo.
}

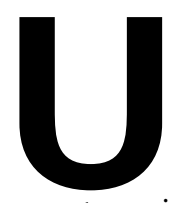
$\mathrm{n}$ des champs les plus actifs et les plus féconds des neurosciences est sans nul doute celui de la neurobiologie du développement, qui tente d'appréhender les mécanismes intervenant dans la construction du cerveau. Il s'agit de comprendre comment l'extraordinaire diversité cellulaire et la complexité de l'organisation des réseaux nerveux, fondée sur la spécificité des innombrables connexions interneuronales, se créent à partir de cellules qui apparaissent précocement dans l'embryon, après quelques divisions répétées de l'œuf fécondé.

D'après Victor Hamburger [1], la neurobiologie du développement moderne prend ses origines, il y a plus d'un siècle (1885-1890), dans la convergence de deux traditions. La première, dite histogénique, est représentée par Wilhelm His et Santiago Ramón y Cajal. Leurs études ont montré que les neurones proviennent de précurseurs cellulaires, les «cellules germinatives» de His, en activité mitotique à la surface interne du tube neural. De plus, chaque fibre nerveuse embryonnaire trouve son origine dans un neurone postmitotique, et elle se termine librement par une structure distale hautement sophistiquée, appelée "cône de croissance». Ces observations constituèrent les premières évidences de l'indépendance morphologique des cellules nerveuses et donc un des fondements de la doctrine neuronale.
La deuxième tradition, celle de la neurobiologie expérimentale, a été conçue par Wilhelm Roux, aussi dans les années 1880. Elle s'appuie sur l'altération de l'intégrité de l'embryon pour tester des hypothèses, et le choix des amphibiens pour réaliser ces expériences a été déterminant. Les expériences réalisées en 1924 par son élève Hans Spemann et par Hilde Mangold pour montrer le processus «d'induction neurale», ont constitué la première grande découverte de la neuroembryologie expérimentale. Spemann et Mangold ont pu prouver que, chez des embryons d'amphibiens, l'ectoderme primitif dans la région dorsale de la blastula ne peut pas se différencier en ébauche du système nerveux si les cellules qui le forment n'établissent pas de contact préalable avec le mésoderme pendant la gastrulation. Le contact ectomésodermique déclenche l'étape de la neurulation, ou formation de la plaque neurale, ébauche de ce qui plus tard deviendra le système nerveux central et périphérique.

Les travaux réalisés entre la fin du XIX siècle et les années 1970, ce que j'appellerais la période classique, ont permis d'établir des notions importantes, par exemple le fait que le développement du cerveau passe par des étapes successives précises. Ainsi, la formation de la plaque neurale est suivie par la neurulation, la fermeture de la plaque donnant naissance au tube neural. Ce dernier va rapide- 
ment acquérir des dilatations espacées, séparées par des constrictions transversales qui lui donnent un aspect segmenté, à l'origine de sa spécification régionale. Les connaissances accumulées pendant cette période classique ont été surtout descriptives, et ne nous ont peu ou pas informés sur les mécanismes mis en jeu. Cependant, les questions essentielles ont été bien posées. Comment I'ectoderme dorsal devient-il tube neural? Comment la prolifération des précurseurs neuronaux est-elle réglée? Comment ces précurseurs vont-ils acquérir leur spécification et leur phénotype? Comment vont-ils migrer et orienter la croissance de leurs axones pour contacter leurs cibles? Comment reconnaissent-ils leurs partenaires spécifiques? Pour quelle raison y a-t-il une mort neuronale si importante et comment meurent ces neurones? Quel est le rôle de I'activité des réseaux émergents dans le raffinement des connexions?

La neurobiologie du développement a réussi, au cours de ces deux dernières décennies, des progrès majeurs, grâce surtout à la révolution technologique de la biologie moléculaire. Nous sommes à présent dans une période nouvelle, dans laquelle le but de ce domaine des neurosciences, comprendre les mécanismes qui sous-tendent l'émergence de la complexité du cerveau, semble enfin à notre portée. II faut rappeler ici que la grande percée de ces dernières années a été surtout due à l'étude de la drosophile et de Caenorhabditis elegans, organismes permettant des analyses génétiques qui ont permis $d^{\prime}$ isoler de nombreux gènes qui interviennent dans le développement du système nerveux. Ensuite, il a été prouvé que ces gènes étaient conservés au cours de l'évolution et que leurs homologues existaient chez les mammifères. Ainsi, la stratégie génétique du développement du cerveau des vertébrés, I'homme inclus, est très similaire à celle employée par les invertébrés. Par exemple, chez la drosophile, c'est une famille de gènes, les gènes $H O M$, qui est responsable du développement de la segmentation antéro-postérieure de l'embryon. La découverte, dans les années 1980, des gènes HOX des vertébrés - homologues des gènes HOM de la drosophile - a permis de bien comprendre le rôle du complexe
HOX dans la métamérisation du rhombencéphale, région correspondant au cerveau postérieur. Ceux-ci, et beaucoup d'autres gènes du développement, ont été identifiés et clonés dans cette période faste de la biologie moléculaire. Certains des gènes clonés n'ont servi que de marqueurs de différenciation, permettant de distinguer des frontières et des compartiments, fournissant des arguments pour montrer qu'à l'égal du cerveau postérieur, le prosencéphale ou cerveau antérieur est aussi segmenté [2]. Plus important, grâce à des techniques de knock-in, on a prouvé que les gènes murins pouvaient remplacer fonctionnellement leurs homologues de la drosophile, et vice versa [3]. Cela indique que les fonctions géniques nécessaires pour le développement du cerveau des mammifères ont évolué à partir d'un ancêtre primitif commun, il y a plus de 500 millions d'années.

Il a été aussi possible d'identifier certains des signaux environnementaux qui contrôlent la transcription des gènes dans les cellules du cerveau, et nous commençons à comprendre les événements post-transcriptionnels responsables de l'identité et de la fonction des cellules nerveuses [4]. Le bilan de ces 20 dernières années est impressionnant. Nous commençons à envisager des réponses à une grande partie des questions héritées de la période classique. Pour ne prendre qu'un exemple - celui des mécanismes moléculaires qui interviennent dans le guidage des neurones en migration et des axones en croissancedes résultats récents montrent la complexité du processus. Outre l'environnement moléculaire dans lequel migrent les neurones et poussent les axones (facteurs locaux), les cellules comme les axones sont également orientés à distance par des molécules sécrétées (chimiotactisme) [5]. Plusieurs familles de molécules chimiotropiques ont été identifiées (nétrines, sémaphorines, slits, etc.) et elles semblent être fonctionnelles aussi bien dans la migration tangentielle des neurones [6] que dans le guidage axonal [7]. Nous connaissons aussi les récepteurs de la transduction du signal donné par ces molécules chimiotropiques et, ce qui complique encore le processus, la majorité de ces molécules peuvent exercer des actions bifonctionnelles (attirantes et répulsives) selon la composition des sites récepteurs. Donc, comme pour d'autres molécules destinées à sculpter le cerveau, il y a conservation phylogénétique, redondance génique et dichotomie fonctionnelle.

Dans un avenir proche, nous aurons à notre disposition les séquences complètes du génome de beaucoup d'invertébrés et de vertébrés, y compris I'homme. Nous entrons ainsi dans la période postgénomique, même $s^{\prime}$ il reste encore beaucoup de gènes à découvrir (par exemple, ceux qui jouent les rôles-clés dans la régulation des processus de prolifération, de différenciation, de synaptogenèse, etc.). Les problèmes qui se posent déjà font dériver les recherches de la neurobiologie du développement vers des études plus physiologiques, destinées à analyser les fonctions des produits des gènes. Il s'agit de comprendre le jeu des protéines codées par les gènes du développement, et surtout la façon dont leur combinatoire d'expression contrôle le développement cérébral. Une façon d'aborder ce problème est I'inactivation génique par recombinaison homologue chez la souris. Cependant la redondance génique, qui caractérise beaucoup de ces processus, apparaît comme une limitation importante de cette approche, et des doubles, voire des triples invalidations seront nécessaires avant de saisir la fonction de nombreux gènes. II faut développer d'autres stratégies, fondées par exemple sur la biologie cellulaire. Beaucoup d'homéogènes ont une double vague d'expression: une première très précoce, destinée à spécifier le tube neural, et une seconde tardive, voire même n'apparaissant que chez I'animal adulte. Quel est le rôle des expressions tardives? II a été suggéré que les protéines à homéo-domaine pourraient avoir une action paracrine ou autocrine, et deviendraient ainsi des signaux gouvernant certaines interactions intercellulaires, orchestrant la morphogenèse du neurone, la croissance axonale et la reconnaissance des cibles [8]. Afin de comprendre leur action physiologique, il convient de caractériser les domaines fonctionnels ainsi que les cibles transcriptionnelles de ces protéines, et de développer des approches in vivo pour l'étude des mécanismes de régulation de leur sécrétion. 
Finalement, il faut rappeler que malgré l'importance des programmes génétiques dans l'ontogenèse du cerveau, des processus aléatoires ou épigénétiques occupent une place de choix, surtout dans les étapes terminales du développement. Cela est le cas pour le raffinement des réseaux neuronaux [9] qui, en augmentant leur précision, permettent le bon fonctionnement du cerveau adulte. Ce raffinement synaptique est le résultat de l'activité coordonnée des ensembles neuronaux préet postsynaptiques, ce qui entraîne un remodelage progressif des arbres terminaux des axones présynaptiques. On pense que les neurotrophines sont les médiateurs de ces processus qui dépendent de l'activité, au moins au niveau du cortex visuel [10]. Il est évident que l'analyse la plus poussée possible doit être effectuée de ces processus qui, à travers les interactions cerveau-monde extérieur, et par des mécanismes dits de plasticité neuronale, vont influencer la spécificité de ces réseaux neuronaux en formation

\section{RÉFÉRENCES (suite)}

6. Bloch-Gallego E, Ezan F, Tessier-Lavigne $M$, Sotelo $C$. Floor plae and netrin-1 are involved in the migration and survival of inferior olivary neurons. J Neurosci 1999; $19: 4407-20$.
7. Nguyen Ba-Charvet K, Brose K, Marillat $\mathrm{V}$, et al. Slit 2-mediated chemorepulsion and collapse of developing forebrain axons. Neuron $1999 ; 22$ : 463-73.

8. Prochiantz A, Théodore L. Homeoproteins, neurite growth and cell recognition. In : Ottoson D, Bartfai T, Hökfelt T, Fuxe K, eds. Challenges and perspectives in neuroscience. Wenner-Gren International Series, vol. 66. Amsterdam: Pergamon-Elsevier Science Ltd, 1995 : 167-78.

9. Changeux JP, Danchin A. Selective stabilisation of developing synapses as a mechanism for the specification of neuronal networks. Nature 1976 ; 264 : 705-12.

10. Berardi N, Maffei L. From visual experience to visual fonction : roles of neurotrophins. J Neurobiol 1999; 41: 119-26.

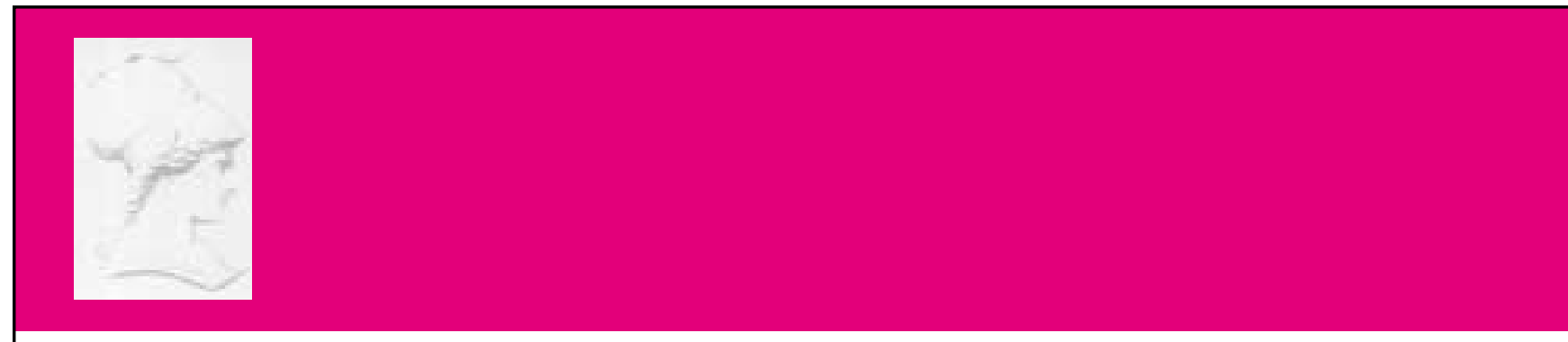

